\title{
Risk of first venous thromboembolism in pregnant women in hospital: population based cohort study from England
}

\author{
(c) (1) (8)
}

\author{
Alyshah Abdul Sultan doctorate student ${ }^{1}$, Joe West associate professor ${ }^{1}$, Laila J Tata associate \\ professor $^{1}$, Kate M Fleming lecturer ${ }^{1}$, Catherine Nelson-Piercy professor ${ }^{2}$, Matthew $\mathrm{J}$ Grainge \\ associate professor ${ }^{1}$
}

'Division of Epidemiology and Public Health, University of Nottingham, Clinical Sciences Building Phase 2, City Hospital, Nottingham NG5 1PB, UK; '²Women's Health Academic Centre, Guy's and St Thomas' Foundation Trust, St Thomas' Hospital, London SE1 7EH, UK

\begin{abstract}
Objective To examine the potential for preventing venous thromboembolism during and after antepartum hospital admissions in pregnant women

Design Cohort study using linked primary (Clinical Practice Research Datalink) and secondary (Hospital Episode Statistics) care records.

Setting Primary and secondary care centres, England.

Participants 206785 women aged 15-44 who had one or more pregnancies from1997 up to 2010.

Main outcome measure Risk of first venous thromboembolism in pregnant women admitted to hospital for one or more days for reasons other than delivery or venous thromboembolism. Risk was assessed by calculating the absolute rate of venous thromboembolism and comparing these rates with those observed during follow-up time not associated with hospital admission using a Poisson regression model to estimate incidence rate ratios.
\end{abstract}

Results Admission to hospital in pregnancy was associated with an increased risk of venous thromboembolism (absolute rate 1752/100 000 person years; incidence rate ratio $17.5,95 \%$ confidence interval 7.69 to 40.0) compared with time outside hospital. The rate of venous thromboembolism was also high during the 28 days after discharge (absolute rate $676 ; 6.27,3.74$ to 10.5). The rate during and after admission combined was highest in the third trimester $(961 ; 5.57,3.32$ to 9.34 ) and in those aged $\geq 35$ years $(1756 ; 21.7,9.62$ to 49.0$)$. While the absolute rate in the combined period was highest for those with three or more days in hospital $(1511 ; 12.2,6.65$ to 22.7$)$, there was also a fourfold increase $(558 ; 4.05,2.23$ to 7.38$)$ in the risk of venous thromboembolism for those admitted to hospital for less than three days.

Conclusion The overall risk of first venous thromboembolism in pregnant women increased during admissions to hospital not related to delivery, and remained significantly higher in the 28 days after discharge. During these periods need for thromboprophylaxis should receive careful consideration.

\section{Introduction}

Venous thromboembolism is one of the leading causes of maternal mortality in developed regions ${ }^{12}$ and is also responsible for many more non-fatal complications such as post-thrombotic syndrome. ${ }^{3}$ Around $50 \%$ of maternal venous thromboembolism events occur during pregnancy, and there is a shortage of high quality evidence regarding which women are at greatest risk. Previous research has shown that obesity, cigarette smoking, multiple gestations, and increased parity are associated with an increased risk of antepartum venous thromboembolism. ${ }^{4-7}$ Of all potential risk factors, however, admission to hospital must be considered as potentially the most important given that in the general population the risk of venous thromboembolism during admission is more than 100 times $^{8}$ the risk outside hospital. Each year in the United Kingdom an estimated 25000 deaths from venous thromboembolism are associated with admissions to hospital. ${ }^{9}$ It is not clear if the same magnitude of risk exists for pregnant women. Also, the risk of venous thromboembolism after discharge needs to be determined to assess the need for thromboprophylaxis after admission.

Current guidelines from the Royal College of Obstetricians and Gynaecologists ${ }^{10}$ recommend that antenatal pharmacological thromboprophylaxis should be considered in pregnant women during admissions to hospital for stays of three or more days if they also have two or more risk factors. Estimates of the absolute risks of venous thromboembolism during and after antepartum admissions, including those in women with no other medical comorbidities or risk factors, however, are not available. Using linked data sources from primary and secondary care we determined the risk of first venous thromboembolism in pregnant 
women admitted to hospital, while accounting for other maternal risk factors and risk factors associated with pregnancy and medical comorbidities.

\section{Method \\ Study population}

We used the Clinical Practice Research Datalink (CPRD), ${ }^{11}$ which is a large longitudinal UK database that contains computerised primary care records of anonymised patients from 1987 onwards. About $98 \%$ of the UK population is registered with general practitioners, who are responsible for nearly all of a patient's medical care. The database includes practices who have been trained to record information with Vision software and who have consented to be included in the database. All patients within a consented practice are automatically included. Around $50 \%$ of the CPRD practices are linked to data from hospital episode statistics (HES) ${ }^{12}$ which contains information on all admissions to hospital in England, including all discharge diagnoses and procedures. The anonymised patient identifiers from CPRD and HES were linked by a trusted third party by using the National Health Service (NHS) number, date of birth, postcode, and sex. ${ }^{13}$ Most patients were matched exactly according to NHS number (over $90 \%$ of patients are linked in this way), with the remaining patients linked probabilistically on the basis of postcode, date of birth, and sex. As HES covers only English hospitals, we excluded practices from Northern Ireland, Wales, and Scotland. Previously data from the linked portion of the CPRD have been shown to be similar in terms of age, sex, and geographical distribution to data from the UK population published by the Office for National Statistics $(\mathrm{ONS})^{14}$ and the findings are generalisable. Both diagnoses of venous thromboembolism ${ }^{15}$ and birth ${ }^{16}$ information in HES maternity data are validated to external sources with reasonable accuracy. We excluded pregnancies of women with previous venous thromboembolism, regardless of whether those events were diagnosed during pregnancy or not. Therefore, when women developed a venous thromboembolism in one pregnancy, we ignored person time resulting from subsequent pregnancies. The study time period was defined from 1997 up to 2010, during which we identified women aged 15-44 registered within CPRD-HES linked practices with no previous venous thromboembolism who had at least one delivery resulting in live or still birth.

\section{Defining pregnancy and associated time period}

Person time for each woman with at least one pregnancy was divided into antepartum (from conception up to two days before childbirth) and "outside pregnancy" (excluding one day before childbirth up to 12 weeks afterwards, which was time associated with the peripartum and postpartum; fig $1 \Downarrow$ ).

\section{Admission to hospital}

For each woman, we obtained information on admissions (other than for delivery and not related to venous thromboembolism) lasting for one or more days. For all admissions that involved a venous thromboembolism event, two investigators (AAS and JW) independently reviewed 30 days of electronic primary and secondary data before and after the event to determine whether the event was the cause or consequence of the admission. Overall, there was good concordance between the investigators $(\kappa=0.8$ ), with disagreement being resolved by consensus. We considered admission to hospital as a time varying covariate that was categorised into three states: "admission" (defined as the time between the date of admission and date of discharge); "post-discharge" (up to 28 days after discharge date), further divided into weeks; and "time outside admission" (time not associated with admission) (fig $1 \Downarrow$ ). Our choice of 28 days for the period after discharge for most analyses was arbitrary but was mainly based on recommendations of thromboprophylaxis among patients undergoing knee surgery. ${ }^{17}$ As such we also ascertained the risk in the period from 28 days to 10 weeks after discharge to assess to what extent the risk in the longer term based on the median duration of follow-up from hospital discharge up till the date of delivery.

\section{Information on other risk factors}

We extracted information on women's demographic factors such as body mass index (BMI) (most recent recording before conception), smoking status (latest recording before the date of delivery), and age (time varying) from their medical records using primary care CPRD data. For each woman, we also extracted information on characteristics associated with the pregnancy (such as multiple gestations) and complications associated with pregnancy (such as hyperemesis, antepartum haemorrhage) using data from both primary and secondary care. We defined women as having gestational diabetes or gestational hypertension if the first medical code for the specified condition (or three or more readings of high blood pressure with systolic $>140 \mathrm{~mm} \mathrm{Hg}$ and/or diastolic $>90 \mathrm{~mm} \mathrm{Hg}$ recorded during pregnancy for gestational hypertension) took place during pregnancy, with no previous medical codes or prescription for antidiabetic or antihypertensive drugs. Those with previous medical codes or drug prescription for diabetes or hypertension were classified as having pre-existing diabetes or hypertension. We investigated common infections (urinary tract infection and acute respiratory tract infections (including pneumonia, acute bronchitis, chest infection, and influenza) recorded during pregnancy (gestational). We also extracted information on other comorbidities that are considered as risk factors in the current RCOG guidelines; specifically cardiac disease, inflammatory bowel disease, nephrotic syndrome, and varicose veins if recorded at any time before the date of delivery.

\section{Venous thromboembolism}

We defined venous thromboembolism as the recording of a medical code relating to the diagnosis of pulmonary embolism or deep vein thrombosis in HES or CPRD where there was evidence of anticoagulation within 90 days of diagnosis or if death occurred within 30 days of diagnosis. Based on this definition $84 \%$ of cases have been previously validated in the CPRD database when only medical codes in primary care were used to define events. ${ }^{15}$

\section{Statistical analysis}

Absolute rates of venous thromboembolism per 100000 person years and $95 \%$ confidence interval were calculated by dividing the total number of events by the person years of follow-up. To determine which factors were associated with an increased risk of venous thromboembolism, we calculated incidence rate ratios for each category of the risk factor for antepartum compared with baseline using a Poisson regression model. Similar methods were used to compare rates of venous thromboembolism during admission and the 28 days after discharge with time outside hospital for antepartum (for comparative purposes this analysis was repeated for "time outside pregnancy"). Initially, we estimated the incidence rate ratio comparing the rate of venous thromboembolism during admission and after discharge to time 
outside hospital adjusted for age and calendar year. The calculated incidence rate ratios were also adjusted for additional risk factors found to be associated with increased risk of venous thromboembolism. Women with missing information on BMI were initially placed in a separate category for purposes of presenting rates of venous thromboembolism according to BMI. For the adjusted analyses of venous thromboembolism rate by admission, we replaced missing values for BMI using multivariate normal imputation. This involved regressing existing BMI values on age and performing analyses on 10 imputed datasets before results were combined. Imputations were carried out using actual BMI values, after which this variable was categorised (with existing BMI categories) before analysis. A sensitivity analysis was also conducted in which we categorised missing BMI as a separate category and included it in our regression analysis. We also fitted a clustering term to take account of multiple pregnancies experienced by a woman. The current RCOG guideline suggests pharmacological thromboprophylaxis for pregnant women admitted to hospital for three or more days with the presence of two or more risk factors including obesity (BMI $>30)$ and any significant comorbidity. Therefore we also calculated the absolute and relative rate for admission and after discharge restricting only to pregnancies in women without those factors.

Subsequent subgroup analyses for antepartum combined the admission and post-discharge periods because of the relatively small number of venous thromboembolism events that occurred in either of these periods. These analyses included stratification of results according to maternal age, BMI, trimester of pregnancy, duration of hospital stay, and calendar year (before and after 2004 based on the publication of first national guideline for thromboprophylaxis during pregnancy). We also formally tested for interaction between maternal age and admission by fitting an interaction term between them and conducting a likelihood ratio test at the $5 \%$ level of significance. Similarly, we tested for an interaction between admission and BMI category using the same methods. All statistical analysis was conducted with Stata version 11.2.

\section{Results}

\section{Basic characteristics}

A total of 206785 women had 245661 pregnancies resulting in live or still birth during the study period. The median follow-up for women in the study was 6.1 years (interquartile range 2.0-10.2). Table 1 shows the basic characteristics of women with those pregnancies $\Downarrow$.

\section{Admission to hospital}

Around $18 \%$ of women's pregnancies (42 256 pregnancies) included at least one admission with 4.7\% (11 472 ) of women admitted more than once during an individual pregnancy. This amounted to a total of 59537 non-delivery periods of admission that occurred during the antepartum period, of which $57 \%$ (33 560 ,), 22\% (12 883), and 22\% (13 094) concluded within one day, two days, and three or more days, respectively. The overall rate of antepartum admission was calculated to be $242 / 1000$ pregnancies. This was much higher in the third trimester (176/1000 pregnancies) than in the first and second trimester (29/1000 and 37/1000 pregnancies, respectively). In total 16 $137(27 \%)$ antepartum periods of admission occurred in women with pre-existing medical risk factors (including cardiac disease, varicose veins, pre-existing diabetes, hypertension, inflammatory bowel disease, and nephrotic syndrome during pregnancy or women with a BMI $\geq 30$ ).

\section{Venous thromboembolism events}

Initially, we identified 100 women who developed venous thromboembolism during or within four weeks after admission (of whom 35 women were pregnant at the time of diagnosis). After the independent reviews, we classed 72 venous thromboembolism events as being a consequence of the admission, of which 46 and 26 occurred during the time outside pregnancy and antepartum period, respectively. There were 176 venous thromboembolism events diagnosed during the antepartum period, of which $15 \%(n=26)$ occurred when the woman was an inpatient (occurring during or up to four weeks after admission and when venous thromboembolism was not the reason of admission).

\section{Rate of venous thromboembolism by maternal risk factors}

Overall the rate of venous thromboembolism during the antepartum period and time "outside pregnancy" was calculated to be $112 / 100000$ and $32 / 100000$ person years, respectively. We found high rates of antepartum venous thromboembolism (more than two fold compared with their respective baseline) in women who had gestational diabetes, gestational acute systemic infection, hyperemesis, varicose veins, or cardiac disease (table $2 \Downarrow$ ).

\section{Rate of venous thromboembolism by admission and after admission}

The risk of antepartum venous thromboembolism increased during admission and after discharge, with absolute rates of $1752 / 100000$ and 676/100 000 person years, respectively (table $3 \Downarrow)$. After adjustment for potential confounding factors (including maternal age and calendar year), there was a 17.5-fold (95\% confidence interval 7.69 to 40.0 ) and a sixfold (3.74 to $10.5)$ increase in risk of venous thromboembolism during admission and after discharge, respectively, compared with outside hospital in the antepartum period. When we considered only admissions in women with no associated pre-existing medical comorbidity or BMI $>30 \mathrm{~kg} / \mathrm{m}^{2}$, the magnitude of the increase in relative risk associated with admission remained similar. When we assessed risk associated with admission for time outside pregnancy, we found that admission and post-discharge corresponded to a 66-fold and a 33-fold increased risk (after adjustment for age and calendar year), respectively, compared with time outside admission (table $3 \Downarrow$ ). For the antepartum period overall, we found the rate of venous thromboembolism altered with duration of hospital stay (table 3 ). Those with a stay of less than three days had four times the risk whereas those with stays of three or more days had over 12 times the risk of venous thromboembolism during admission/after discharge compared with time outside hospital in the antepartum period.

\section{Variation of the risk by age, trimester, BMI, and calendar year}

We also found the collective rate of venous thromboembolism during admission and after discharge to be higher in the third trimester of pregnancy (absolute rate $961 / 100000$ person years; table $4 \Downarrow$ ). The rate increased with age, with the highest risk among women aged $\geq 35$ (1756/100 000 person years). Furthermore, we observed a significant interaction between age and admission $(\mathrm{P}=0.01)$. Our results highlighted that the increased risk associated with admission and after discharge remains broadly unaltered by women's BMI, as supported by a non-significant test result for interaction between BMI 
category and admission $(\mathrm{P}=0.36)$. We also observed higher absolute and relative rates of venous thromboembolism after 2004 during admission and after discharge. All our calculated incidence rate ratios remained broadly similar when we additionally adjusted for other risk factors significantly associated with increased risk of venous thromboembolism (model 2).

\section{Rate of venous thromboembolism in weeks after hospital admission}

The rate of venous thromboembolism after admission was highest during the first two weeks after discharge (763/100 000 person years; incidence rate ratio $7.98,95 \%$ confidence interval 4.50 to 14.1 ) (fig $2 \Downarrow$ ). The risk decreased after four weeks, with an absolute rate of $387 / 100000$ person years $(4.19,4.57$ to 11.6$)$ between five and 10 weeks after discharge.

\section{Discussion}

\section{Main findings}

Using nationally linked primary and secondary care data, we have provided population level absolute and relative rates of antepartum venous thromboembolism by admission to hospital (including after discharge) while taking into account other risk factors for venous thromboembolism. We found that $18 \%$ of pregnant women were admitted to hospital at least once during pregnancy (before the delivery admission). Most admissions were of short duration and occurred during the third trimester. While $85 \%$ of antepartum venous thromboembolism events occurred in outpatients, the risk was 17 -fold higher during admission to hospital compared with time "outside hospital." We also found a higher rate of venous thromboembolism during the first 28 days after discharge, corresponding to a six fold risk compared with baseline. While the rate of venous thromboembolism during admission and after discharge was particularly high for women with stays of three or more days, there was also a fourfold increase in the risk of venous thromboembolism for those admitted to hospital for less than three days. The rate for admission/after discharge was also high in all three trimesters and in women aged $\geq 35$ years. The association between admission and venous thromboembolism remained when we restricted our analysis to women without medical comorbidities including obesity, cardiac disease, and varicose veins.

\section{Strengths and limitations}

Our study used an open cohort approach, with prospectively collected data and information from linked primary and secondary care data sources from all over England, covering $3 \%$ of the total UK population with a similar age and sex distribution to the population as a whole..$^{14}$ Our finding should be generalisable to the UK and other developed nations with similar healthcare systems. The use of linked data enabled us not only to obtain comprehensive information on risk factors documented in either level of care (primary and secondary) but gave us the opportunity to follow pregnant women in and outside hospital with accurate dates of admission and discharge. This permitted us to calculate the absolute and relative rates of antepartum venous thromboembolism with respect to admission while taking into account other potential risk factors, which has not been assessed previously in the literature. The completeness of HES data and recording of diagnoses in different centres is always a concern. The Department of Health, however, has undertaken studies to assess the completeness of HES coverage, which is reported to be high. ${ }^{18}$ Moreover, a systematic review of discharge coding in HES found that the median coding accuracy rate for diagnostic codes is $91 \% .{ }^{19}$ As we used data from both primary and secondary care, our prevalence of risk factors such as diabetes in pregnancy, gestational hypertension, and antepartum haemorrhage is similar to the expected prevalence in the $\mathrm{UK} .^{20-23}$

A limitation of this study is the relatively small number of venous thromboembolism events either during or immediately after admission. This not only gave us estimates with wide confidence intervals but restricted our ability to stratify our analysis by reason for admission. We also acknowledge that the increased risk of venous thromboembolism during admission could be caused by other well understood risk factors (such as unmeasured comorbidities), leading to an overestimate in the independent effect of admission. Our calculated estimates for admission and after discharge, however, remained unchanged when we excluded pregnant women with other known risk factors. We also found that the increased rate of venous thromboembolism decreased in the weeks after discharge, which should not have been the case if the effect was solely because of other risk factors. Despite the fact that the risk of venous thromboembolism was high in the later period after discharge (five to 10 weeks), the decrease in the risk from around 1800 (during admission) to 387 per 100000 person years shows some longer term effect of admission on the incidence of venous thromboembolism. While the cause of the association between admission and venous thromboembolism is not clear, immobility is often considered the main culprit. ${ }^{4}$ Regardless of the reasons for admission, pregnant women admitted to hospital for reasons other than delivery represent a group at high risk as evident by a fourfold increased risk in those with stays in hospital of less than three days.

Our conclusions, in part, depend on two investigators agreeing on whether the venous thromboembolism was a cause or consequence of the hospital admission. To determine this, two of the investigators carefully and independently reviewed 30 days of electronic primary and secondary data before and after all venous thromboembolisms during the antepartum period. Overall, agreement between investigators was high. When we repeated analyses firstly accepting only cases when investigator 1 judged antepartum venous thromboembolism to be the consequence of the admission ( $\mathrm{n}=24$ events) and secondly accepting only cases judged by investigator 2 to be a consequence ( $n=27$ events), the impact on our effect estimates was modest and our conclusions were unaltered on both occasions. Subsequently we also involved a third investigator, who independently agreed with the consensus assessment of the other investigators for all cases.

We acknowledge that the diagnosis of venous thromboembolism in pregnancy can be difficult and that leg swelling and calf pain are common in the third trimester in women without deep vein thrombosis. This could lead to a misclassification. Additionally, D-dimer levels also increase with gestation, ${ }^{2}$ with gestational hypertension, and in preterm labour, leading to false positive results if normal ranges for non-pregnant women are use, which could add to this misclassification. In pregnancy, however, venous thromboembolism is diagnosed only when there is confirmatory imaging, and for our analysis we accepted only a diagnosis made by a clinician if it was complemented by use of anticoagulant therapy. One current drawback of UK secondary care data is the lack of information on heparin and warfarin prescribed in hospital, which might have led to underascertainment of cases using our definition of venous thromboembolism. We believe, however, that the impact of this limitation should be minimal as pregnant women with a 
diagnosis of venous thromboembolism are expected to be given anticoagulation therapy throughout the remainder of their pregnancy. ${ }^{24}$ Therefore these prescriptions are likely to be captured in the primary care data. Furthermore, the algorithm used to define venous thromboembolism in our study has been previously validated in primary care data with a positive predicted value of $85 \%,{ }^{15}$ and our estimates of the incidence of venous thromboembolism during the antepartum period are in concordance with most recent studies published on this topic. ${ }^{25}$

There could have been under ascertainment of deep vein thrombosis during the time outside hospital compared with during admission, when women would be closely monitored for venous thromboembolism thus inflating our rate ratios for that period. We believe venous thromboembolism events occurring outside hospital, however, will be captured in primary care data with a similar degree of ascertainment as pregnancy is a known and established risk factor. It also is worth stating that we were not able to consider certain risk factors such as thrombophilia and anti-phospholipid syndrome, which are often associated with increased risk. We believe that pragmatically those risk factors cannot be used to predict venous thromboembolism as routine screening for thrombophilia/anti-phospholipid syndrome is not recommended for pregnant women. Additionally the impact of those variables on our estimates might be small as we considered only the first venous thromboembolism whereas most cases of thrombophilia may be diagnosed after a venous thromboembolism event has occurred.

Our increased absolute risk observed during admission and after discharge to a certain extent advocates the use of thromboprophylaxis measures during that period. Though it could be argued that more liberal use of heparin might be associated with a higher risk of osteoporosis, it should be noted that low molecular weight heparin is now commonly used for thromboprophylaxis during pregnancy and is considered to be safe and effective. For instance, a systematic review ${ }^{26}$ of 2777 pregnancies reported no increased risk of heparin induced thrombocytopenia or osteoporosis associated with use of low molecular weight heparin.

Our estimates for risk of venous thromboembolism during admission and after discharge do not take into account that some pregnant women might already be receiving thromboprophylaxis during those periods. We believe, however, that since the first RCOG guidelines for antenatal thromboprophylaxis were published only in 2004 (updated 2009), the use of antenatal thromboprophylaxis with low molecular weight heparin was unusual before 2004 except for those women with previous venous thromboembolism. We found that both absolute and relative rates of venous thromboembolism have increased since 2004, which should not be the case if those women were given adequate pharmacological thromboprophylaxis. This does not take into account the increasing ascertainment of less severe venous thromboembolisms. Additionally, $67 \%$ of all pregnant women diagnosed with antepartum pulmonary embolism (between 2005 and 2006) in the UK did not receive pharmacological thromboprophylaxis according to national guidelines even though they qualified for thromboprophylaxis. ${ }^{5}$ Despite these limitations, we believe a bespoke cohort study of a similar size and scale to our study (using routine data) in which women are followed up prospectively throughout pregnancy would not be practically possible.

Finally in this study we have provided absolute risk expressed as per 100000 person years, which helps standardisation and comparison. Another way of expressing those estimates might be as risk per admission. For instance, 26 venous thromboembolism events out of 59537 hospital admissions gives an overall rate of one per 2000 hospital admissions and one per 1000 for those admitted for longer duration (three days and more). Though this might be of potential clinical use, it should be noted that these calculations are crude and do not take into account the element of time associated with each hospital episode.

\section{Comparison with previous studies}

To our knowledge this is the first study to assess the impact of antepartum admission to hospital on the incidence of venous thromboembolism during pregnancy. Therefore we cannot directly compare our findings with those from other research. A previous study carried out in Rochester, Minnesota, however, showed an age adjusted 135 -fold $^{8}$ increased risk of venous thromboembolism among patients in hospital compared with people outside hospital in the general population (including men); in the present study to observed increase outside pregnancy was 67 -fold. While our confidence interval for this estimate (36.3 to 120) does not include the value observed in the Rochester study, this might not be surprising given that the Rochester study was carried out in a much older population (mean age 65), who would therefore be more likely to have longer inpatient spells complicated by comorbidity .

\section{Clinical implications}

We believe that this study has important implications in the way pharmacological thromboprophylaxis is delivered to pregnant women and hope that it will help targeting prophylaxis in three ways. Firstly, we found a six fold increased risk of venous thromboembolism in the 28 days after hospital discharge. This suggests prudent consideration of all pregnant women during that period in terms of assessment of risk of venous thromboembolism. Secondly, at present RCOG guidelines advise that prophylaxis should be considered for women at the time of hospital admission provided that she has two or more risk factors including obesity (BMI >30) and significant medical comorbidity and is expected to be immobile for three or more days. Our study showed that the risk of venous thromboembolism during admission and after discharge remained around 22-fold and eightfold, respectively, even in women without such risk factors. Therefore pregnant women might be at high risk of venous thromboembolism during admission and for 28 days after discharge, particularly those with longer stay in hospital. Our post hoc analysis found a higher risk of venous thromboembolism among pregnant women aged $\geq 35$ years admitted to hospital. This should be investigated further in future research. Finally, the risk of venous thromboembolism remained increased fourfold (during admission and 28 days after discharge) even in women who stay in hospital for less than three days (rate equivalent to $0.4 \%$ per year), which might also require careful consideration. It is important to take into account the effectiveness of low molecular weight heparin in term of costs involved in prophylaxis both financial and also the tolerability surrounding a daily heparin prescription as well as the well recognised side effects of allergy and bleeding. For instance, the benefits would need to be weighed against a risk of major haemorrhage, which is believed to occur in $1 \%$ of pregnant women. Such a risk:benefit analysis clearly goes beyond the scope of the present work; however, we believe our presentation of population based risks of venous thromboembolism in pregnant women admitted to hospital goes some way to help clinicians involved in making decisions in this area. In conclusion, we found that admission substantially increases the risk of venous thromboembolism in pregnant 
women as has been found in other non-pregnant populations. These findings support the National Institute of Clinical Excellence guidelines on thromboprophylaxis among admitted patients. ${ }^{27}$ In light of this, careful consideration of which women should receive prophylaxis during an antepartum admission and for how long is needed.

Contributors: AAS, JW, and MJG conceived the idea for the study, with LJT and KMF also making important contributions to the design. AAS carried out the data management and analysis and wrote the first draft of the manuscript. CNP provided clinical input and interpretation at all stages of the project. All authors were involved in the interpretation of the data, contributed towards critical revision of the manuscript and approved the final draft. AAS had full access to all of the data and MJG had final responsibility for the decision to submit for publication. AAS and MJG are guarantors.

Funding: AAS is funded by a scholarship awarded by the Aga Khan Foundation. JW is funded by a University of Nottingham senior clinical research fellowship, which also contributes to AAS's funding.

Competing interests: All authors have completed the ICMJE uniform disclosure form at www.icmje.org/coi_disclosure.pdf and declare: CNP was co-developer of the currently available guidelines on VTE prophylaxis in pregnancy issued by the Royal College of Obstetricians and Gynaecologists (green top guideline 37a). CNP has also received honorariums for lectures from Leo Pharma and Sanofi Aventis (makers of tinzaparin and enoxaparin, low molecular weight heparins used in obstetric thromboprophylaxis) and has received payment from Leo Pharma for development of an educational "slide kit" about obstetric thromboprophylaxis.

Ethical approval: This study was reviewed and approved by CPRD Independent Scientific Advisory Committee (ISAC) Ref No 10_193R.

Declaration of transparency: AAS and MJG affirm that the manuscript is an honest, accurate, and transparent account of the study being reported; that no important aspects of the study have been omitted; and that any discrepancies from the study as planned (and, if relevant, registered) have been explained.

Data sharing: no additional data available

1 Saving Mothers' Lives: Reviewing maternal deaths to make motherhood safer: 2006-2008. Br J Obetet Gynaecol 2011;118:1-203.

2 Drife J. Thromboembolism. Br Med Bull 2003;67:177-90.

3 Wik H, Jacobsen A, Sandvik L, Sandset P. Prevalence and predictors for post-thrombotic syndrome 3 to 16 years after pregnancy-related venous thrombosis: a population-based, cross-sectional, case-control study. J Thromb Haemost 2012;10:840-7.

4 Jacobsen AF, Skjeldestad FE, Sandset PM. Ante- and postnatal risk factors of venous thrombosis: a hospital-based case-control study. J Thromb Haemost 2008;6:905-12.

5 Knight M. Antenatal pulmonary embolism: risk factors, management and outcomes. Br J Obstet Gynaecol 2008;115:453-61.
6 Larsen TB, Sorensen HT, Gislum M, Johnsen SP. Maternal smoking, obesity, and risk of venous thromboembolism during pregnancy and the puerperium: a population-based nested case-control study. Thromb Res 2007;120:505-9.

7 Simpson EL, Lawrenson RA, Nightingale AL, Farmer RD. Venous thromboembolism in pregnancy and the puerperium: incidence and additional risk factors from a London perinatal database. Br J Obstet Gynaecol 2001;108:56-60.

8 Heit JA, Melton LJ, Lohse CM, Petterson TM, Silverstein MD, Mohr DN, et al. Incidence of venous thromboembolism in hospitalized patients vs community residents. Mayo Clin Proc 2001;76:1102-10.

9 Department of Health. Venous thromboembolism prevention: a patient safety priority (19/04/2013). www.kingsthrombosiscentre.org.uk/kings/12.pdf.

10 Royal College of Obstetricians and Gynaecologists. Reducing the risk of thrombosis and embolism during pregnancy and the puerperium. Green-top Guideline No. 37a 2009.

11 Clinical Practice Research Database. (19/04/2013). www.cprd.com/intro.asp.

12 Hospital Episode Statistics. (19/04/2013). www.hesonline.nhs.uk.

13 Eaton SC, Williams TJ, Puri S, VanStaa T. The feasibility of linking the English Hospital Episode Statistics to the GPRD. Pharmacoepidemiol Drug Saf 2008;17:S214.

14 Crooks C. Epidemiology of upper gastrointestinal bleeding studying its causes and outcomes using case control studies and surivival analyses. [PhD thesis]. University of Nottingham 2013.

15 Lawrenson R, Todd JC, Leydon GM, Williams TJ, Farmer RD. Validation of the diagnosis of venous thromboembolism in general practice database studies. Br J Clin Pharmacol 2000;49:591-6.

16 Dattani N, Datta-Nemdharry P, Macfarlane A. Linking maternity data for England 2007: methods and data quality. Health Stat Q 2012;53.

17 Geerts WH, Bergqvist D, Pineo GF, Heit JA, Samama CM, Lassen MR, et al. Prevention of venous thromboembolism*: American College of Chest Physicians Evidence-Based Clinical Practice Guidelines. Chest 2008;133(6 suppl):381-453S.

18 Thompson A, Shaw M, Harrison G, Ho D, Gunnell D, Verne J. Patterns of hospital admission for adult psychiatric illness in England: analysis of Hospital Episode Statistics data. Br J Psychiatry 2004;185:334-41.

19 Campbell SE, Campbell MK, Grimshaw JM, Walker AE. A systematic review of discharge coding accuracy. J Public Health 2001;23:205-11.

20 National Institute for Health and Clinical Excellence. Diabtes in pregnancy: Management of diabetes and its complication from pre-conception to postnatal period. RCOG Press, 2008.

21 National Intitute for Health and Clinical Excellence. Diabetes in pregnancy: Management of diabetes and its complications from pre-conception to the postnatal period. NICE, 2008.

22 Walker RL, Hemmelgarn B, Quan $\mathrm{H}$. Incidence of gestational hypertension in the Calgary Health Region from 1995 to 2004. Can J Cardiol 2009;25:e284-7.

23 Zhang J, Zeisler J, Hatch MC, Berkowitz G. Epidemiology of pregnancy-induced hypertension. Epidemiol Rev 1997;19:218.

24 Royal College of Obstetricians and Gynaecologist. The acute management of thrombosis and embolism during pregnancy and puerperium. Green-top guideline No. 37b. RCOG Press, 2010.

25 Abdul Sultan A, Tata LJ, Grainge MJ, West J. The incidence of first venous thromboembolism in and around pregnancy using linked primary and secondary care data: a population based cohort study from england and comparative meta-analysis. Plos One 2013;8:e70310.

26 Greer IA, Nelson-Piercy C. Low-molecular-weight heparins for thromboprophylaxis and treatment of venous thromboembolism in pregnancy: a systematic review of safety and efficacy. Blood 2005;106:401-7.

27 National Institute for Health and Clinical Excellence. Venous thromboembolism: reducing the risk of venous thromboembolism (deep vein thrombosis and pulmonary embolism) in patients admitted to hospital. No. 92. NICE Publisher, 2010.

Accepted: 23 September 2013

\section{Cite this as: BMJ 2013;347:f6099}

This is an Open Access article distributed in accordance with the Creative Commons Attribution Non Commercial (CC BY-NC 3.0) license, which permits others to distribute, remix, adapt, build upon this work non-commercially, and license their derivative works on different terms, provided the original work is properly cited and the use is non-commercial. See: http://creativecommons.org/licenses/by-nc/3.0/. 


\section{What is already known on the topic}

Venous thromboembolism, which complicates one to two pregnancies in every 1000 , is one of the leading direct causes of maternal deaths in developed countries

Hospital admission is known to increase the risk of venous thromboembolism about 100-fold, and is responsible for more than 25000 deaths each year in the United Kingdom

The magnitude of risk of venous thromboembolism during antepartum admission and after discharge in terms of absolute risk is unknown

\section{What this paper adds}

The study provides population based absolute and relative rates of venous thromboembolism during antepartum admission and after discharge by using linked primary and secondary care data

There was a high relative rate of venous thromboembolism during inpatient admissions and in the 28 days after discharge, with a particularly high rate among women admitted for three or more days

\section{Tables}

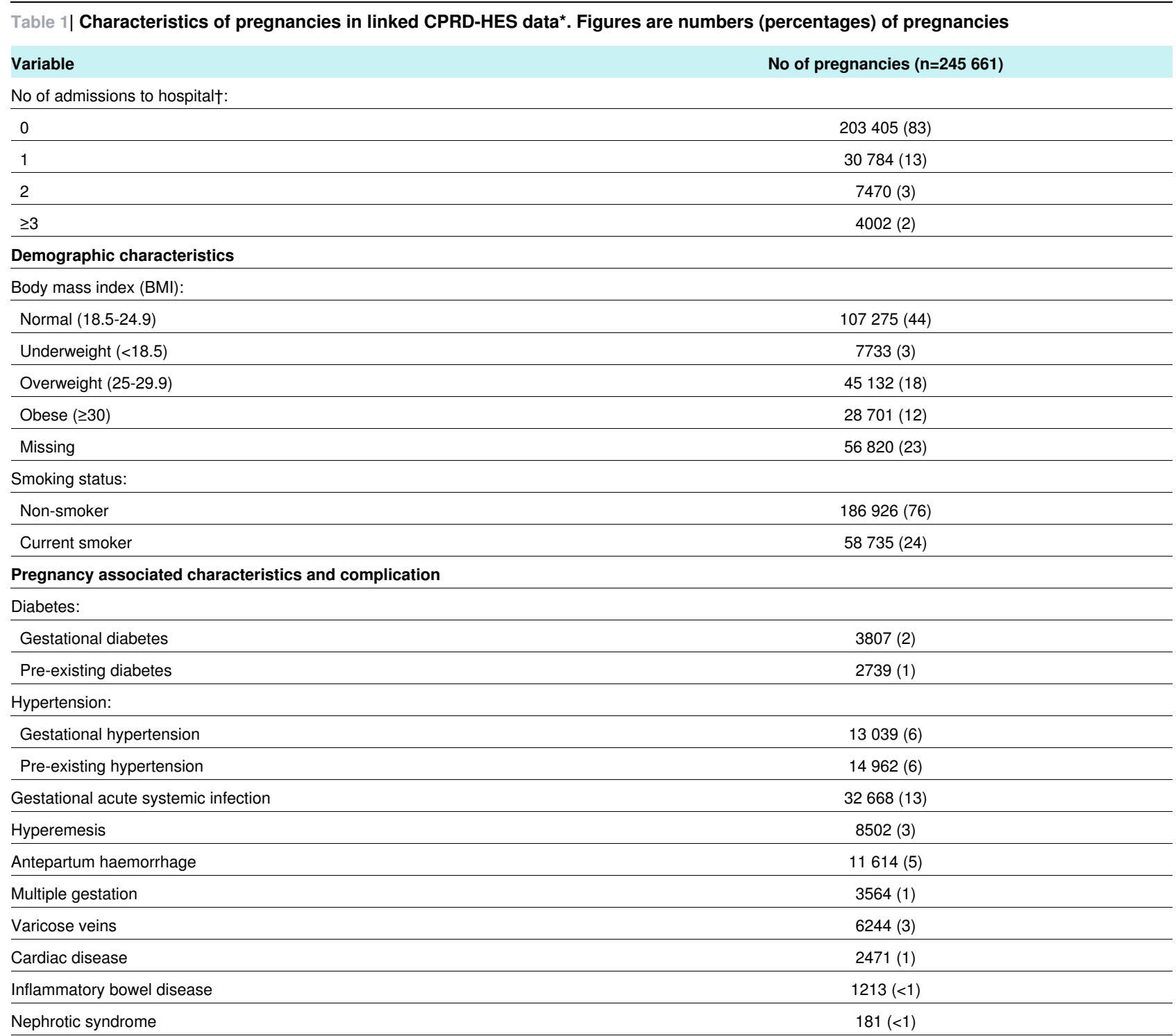

*Pregnancies in 206785 individual women. When women had more than one pregnancy, status of all above risk factors could potentially differ between pregnancies. †During antepartum period. 
Table 2| Absolute rate of venous thromboembolism (VTE) per 100000 person years and incidence rate ratios (IRR) during antepartum period by potential risk factors

\begin{tabular}{|c|c|c|c|}
\hline Variable & No of VTE & Rate* $^{*}(95 \% \mathrm{Cl})$ & IRR (95\% CI) (unadjusted) \\
\hline \multicolumn{4}{|l|}{ Demographic characteristics } \\
\hline \multicolumn{4}{|l|}{ Maternal age (years): } \\
\hline $15-24$ & 38 & 101 (74 to 139$)$ & 0.89 (0.61 to 1.29$)$ \\
\hline $25-34$ & 103 & 114 (94 to 138$)$ & 1.00 \\
\hline $35-44$ & 35 & $118(85$ to 164$)$ & $1.03(0.70$ to 1.52$)$ \\
\hline \multicolumn{4}{|l|}{ Body mass index (BMI): } \\
\hline Normal (18.5-24.9) & 61 & 85 (66 to 110$)$ & 1.00 \\
\hline Underweight $(<18.5)$ & 3 & 59 (19 to 185$)$ & $0.85(0.64$ to 2.09$) \dagger$ \\
\hline Overweight (25-29.9) & 37 & 120 (87 to 166$)$ & $1.29(0.85$ to 1.96$) \dagger$ \\
\hline Obese $(\geq 30)$ & 35 & 178 (128 to 248$)$ & $1.76(1.16$ to 2.67$) \dagger$ \\
\hline Missing & 40 & 130 (95 to 177$)$ & NA† \\
\hline \multicolumn{4}{|l|}{ Smoking status: } \\
\hline Non-smoker & 128 & 107 (90 to 127$)$ & 1.00 \\
\hline Current smoker & 48 & 127 (96 to 169$)$ & $1.18(0.85$ to 1.65$)$ \\
\hline \multicolumn{4}{|l|}{ Antepartum complicationsł } \\
\hline Hyperemesis & 11 & 222 (123 to 402$)$ & 2.05 (1.16 to 3.78$)$ \\
\hline Antepartum haemorrhage & 4 & 181 (67 to 482$)$ & $1.63(0.60$ to 4.39$)$ \\
\hline Multiple gestation & 5 & 223 (93 to 537 ) & 2.02 (0.83 to 4.92$)$ \\
\hline Gestational diabetes & 9 & 361 (187 to 694 ) & 3.30 (1.69 to 6.46$)$ \\
\hline Gestational systemic infection & 30 & 269 (188 to 358$)$ & 2.69 (1.81 to 3.98$)$ \\
\hline Gestational hypertension & 14 & 170 (100 to 287$)$ & 1.63 (0.94 to 2.83$)$ \\
\hline \multicolumn{4}{|l|}{ Medical comorbiditiesł } \\
\hline Varicose veins & 11 & 253 (140 to 458$)$ & 2.35 (1.27 to 4.32$)$ \\
\hline Cardiac disease & 5 & 303 (126 to 728$)$ & $2.75(1.13$ to 6.70$)$ \\
\hline Pre-existing hypertension & 18 & 174 (110 to 277$)$ & 1.64 (1.03 to 2.74$)$ \\
\hline
\end{tabular}

${ }^{*}$ Rate per 100000 person years.

†Missing data imputed.

ҒIRR compared with pregnancies without condition under study. Note: no VTE events were observed in pregnancies complicated by inflammatory bowel disease, nephrotic syndrome, or pre-existing diabetes during antepartum period. 


\begin{tabular}{|c|c|c|c|c|}
\hline \multirow[b]{2}{*}{ Variable } & \multirow[b]{2}{*}{ No of VTE } & \multirow[b]{2}{*}{ Rate $^{*}(95 \% \mathrm{Cl})$} & \multicolumn{2}{|c|}{ IRR (95\% Cl) } \\
\hline & & & Adjusted 1† & Adjusted 2‡ \\
\hline \multicolumn{5}{|l|}{ Antepartum overall } \\
\hline Time outside hospital & 150 & 97 (83 to 114$)$ & 1.00 & 1.00 \\
\hline Hospital admission & 6 & 1752 (787 to 3900$)$ & $18.2(8.04$ to 41.3$)$ & 17.5 (7.69 to 40.0$)$ \\
\hline After discharge & 20 & 676 (436 to 1048 ) & $7.08(4.41$ to 11.3$)$ & 6.27 (3.74 to 10.5$)$ \\
\hline \multicolumn{5}{|c|}{ Pregnancies not complicated by BMI $>30$ or major medical comorbidity } \\
\hline Time outside hospital & 109 & 84 (69 to 101$)$ & 1.00 & - \\
\hline Hospital admission & 5 & 1821 (757 to 4375$)$ & $22.0(8.97$ to 54.3$)$ & - \\
\hline After discharge & 15 & $623(375$ to 1034$)$ & 7.56 (4.36 to 13.1$)$ & - \\
\hline \multicolumn{5}{|c|}{ Variation by duration of hospital stay (combining admission/after discharge) } \\
\hline \multicolumn{5}{|l|}{ Antepartum overall: } \\
\hline Time outside hospital & 150 & 97 (83 to 114$)$ & 1.00 & 1.00 \\
\hline$<3$ days & 13 & 558 (331 to 943$)$ & 5.85 (3.37 to 10.1$)$ & 4.05 (2.23 to 7.38$)$ \\
\hline$\geq 3$ days & 13 & 1511 (858 to 2661 ) & 15.7 (8.71 to 28.5$)$ & 12.2 (6.65 to 22.7 ) \\
\hline \multicolumn{5}{|c|}{ Time outside pregnancy } \\
\hline Time outside hospital & 326 & 28 (25 to 31$)$ & 1.00 & - \\
\hline Hospital admission & 11 & 1890 (1046 to 3412 ) & 66.2 (36.3 to 120$)$ & - \\
\hline After discharge & 35 & 911 (654 to 1269 ) & 32.3 (22.8 to 45.8$)$ & - \\
\hline
\end{tabular}

IRR=incidence rate ratio.

*Rate calculated per 100000 person years.

†Adjusted for maternal age and calendar year when not stratified by them.

$\ddagger$ Adjusted for maternal age, calendaryear, BMI, gestational infection, cardiac disease, varicose vein, gestational diabetes, and hyperemesis.

§Including varicose vein and cardiac disease. 
Table 4| Rate of first antepartum venous thromboembolism (VTE) during hospital admission/after discharge stratified by trimester, age, calendar year and BMI

\begin{tabular}{|c|c|c|c|c|}
\hline \multirow[b]{2}{*}{ Variable } & \multirow[b]{2}{*}{ No of VTE } & \multirow[b]{2}{*}{ Rate $^{*}(95 \% \mathrm{Cl})$} & \multicolumn{2}{|c|}{ IRR (95\% Cl) } \\
\hline & & & Adjusted 1† & Adjusted 2‡ \\
\hline \multicolumn{5}{|c|}{ Variation by antepartum trimester } \\
\hline \multicolumn{5}{|l|}{ Trimester 1 and 2: } \\
\hline Time outside hospital & 60 & 60 (46 to 77$)$ & 1.00 & 1.00 \\
\hline Admission/after discharge & 5 & 449 (186 to 1078$)$ & 7.61 (3.03 to 19.0$)$ & 8.43 (3.27 to 21.7 ) \\
\hline \multicolumn{5}{|l|}{ Trimester 3: } \\
\hline Time outside hospital & 90 & $162(134$ to 203$)$ & 1.00 & 1.00 \\
\hline Admission/after discharge & 21 & 961 (426 to 1473$)$ & 5.93 (3.64 to 9.65$)$ & 5.57 (3.32 to 9.34$)$ \\
\hline \multicolumn{5}{|c|}{ Variation by calendar year in admission/after discharge } \\
\hline \multicolumn{5}{|l|}{ 1997-2003: } \\
\hline Time outside hospital & 62 & 96 (74 to 123$)$ & 1.00 & 1.00 \\
\hline Admission/after discharge & 8 & 550 (275 to 1100$)$ & $5.82(2.76$ to 12.2$)$ & $4.59(2.08$ to 10.1$)$ \\
\hline \multicolumn{5}{|l|}{ 2004-10: } \\
\hline Time outside hospital & 88 & 98 (79 to 121$)$ & 1.00 & 1.00 \\
\hline Admission/after discharge & 18 & 975 (614 to 1548$)$ & $10.1(6.03$ to 16.9$)$ & 8.51 (4.94 to 14.6$)$ \\
\hline \multicolumn{5}{|c|}{ Variation by maternal age (years) } \\
\hline \multicolumn{5}{|c|}{ 15-24: } \\
\hline Time outside hospital & 34 & 93 (66 to 131$)$ & 1.00 & 1.00 \\
\hline Admission/after discharge & 4 & 363 (136 to 967$)$ & $3.88(1.37$ to 10.9$)$ & 3.80 (1.25 to 11.5$)$ \\
\hline \multicolumn{5}{|l|}{ 25-34: } \\
\hline Time outside hospital & 90 & 101 (82 to 124$)$ & 1.00 & 1.00 \\
\hline Admission/after discharge & 13 & 771 (447 to 1328$)$ & 7.61 (4.24 to 13.6$)$ & 6.15 (3.24 to 11.7$)$ \\
\hline \multicolumn{5}{|l|}{ 35-44: } \\
\hline Time outside hospital & 26 & 89 (60 to 131$)$ & 1.00 & 1.00 \\
\hline Admission/after discharge & 9 & 1756 (913 to 3376$)$ & $19.6(9.20$ to 42.0$)$ & 21.7 (9.62 to 49.0$)$ \\
\hline \multicolumn{5}{|l|}{ Variation by BMI } \\
\hline \multicolumn{5}{|l|}{ Normal BMI (18.5-24.9) } \\
\hline Time outside hospital & 51 & 72 (55 to 95$)$ & 1.00 & 1.00 \\
\hline Admission/after discharge & 10 & 766 (412 to 1423$)$ & $6.24(2.41$ to 16.1$)$ & $4.72(1.71$ to 13.0$)$ \\
\hline \multicolumn{5}{|l|}{ Overweight (25-29.9): } \\
\hline Time outside hospital & 34 & $113(81$ to 158$)$ & 1.00 & 1.00 \\
\hline Admission/after discharge & 3 & 474 (153 to 1472$)$ & 10.3 (5.15 to 20.8$)$ & 9.42 (4.38 to 20.5$)$ \\
\hline \multicolumn{5}{|l|}{ Obese $(\geq 30)$ : } \\
\hline Time outside hospital & 30 & 157 (109 to 224$)$ & 1.00 & 1.00 \\
\hline Admission/after discharge & 5 & 974 (405 to 2341$)$ & $4.30(1.30$ to 14.1$)$ & 4.50 (1.23 to 16.4$)$ \\
\hline
\end{tabular}

\section{IRR=incidence rate ratio.}

*Rate calculated per 100000 person years.

†Adjusted for maternal age and calendar year when not stratified by them.

¥Adjusted for maternal age, calendaryear, BMI, gestational infection, cardiac disease, varicose vein, gestational diabetes, and hyperemesis when not stratified by them. 


\section{Figures}

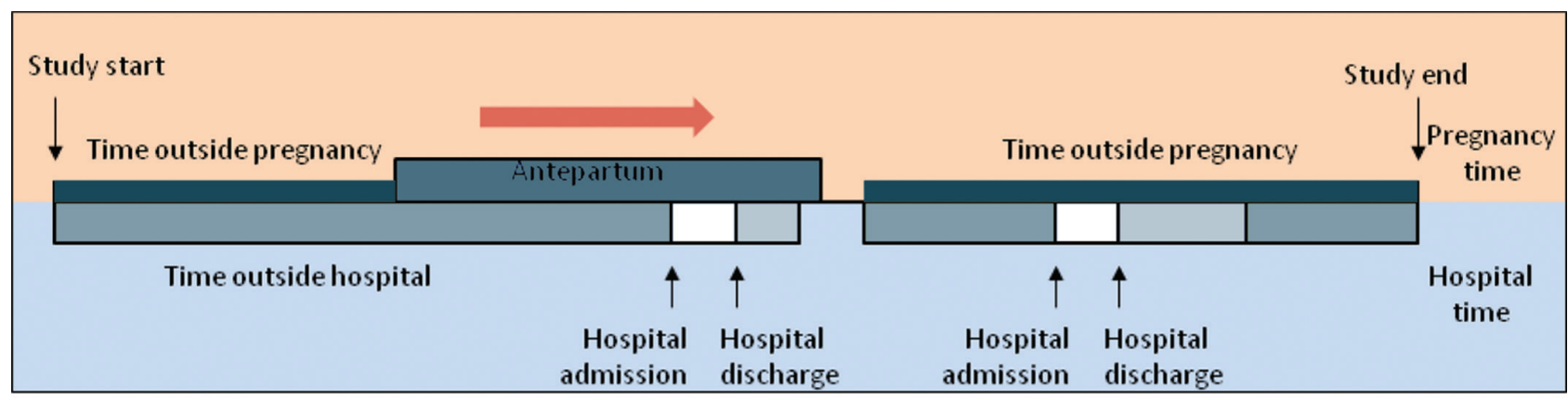

$\square$ Hospitalisation period $\square$ Post-discharge period $\square$ Time outsidehospitalisation $\square$ Antepartumperiod

Time outside pregnancy

Fig 1 Division of antepartum person time into "hospitalised time" and "time outside hospital"

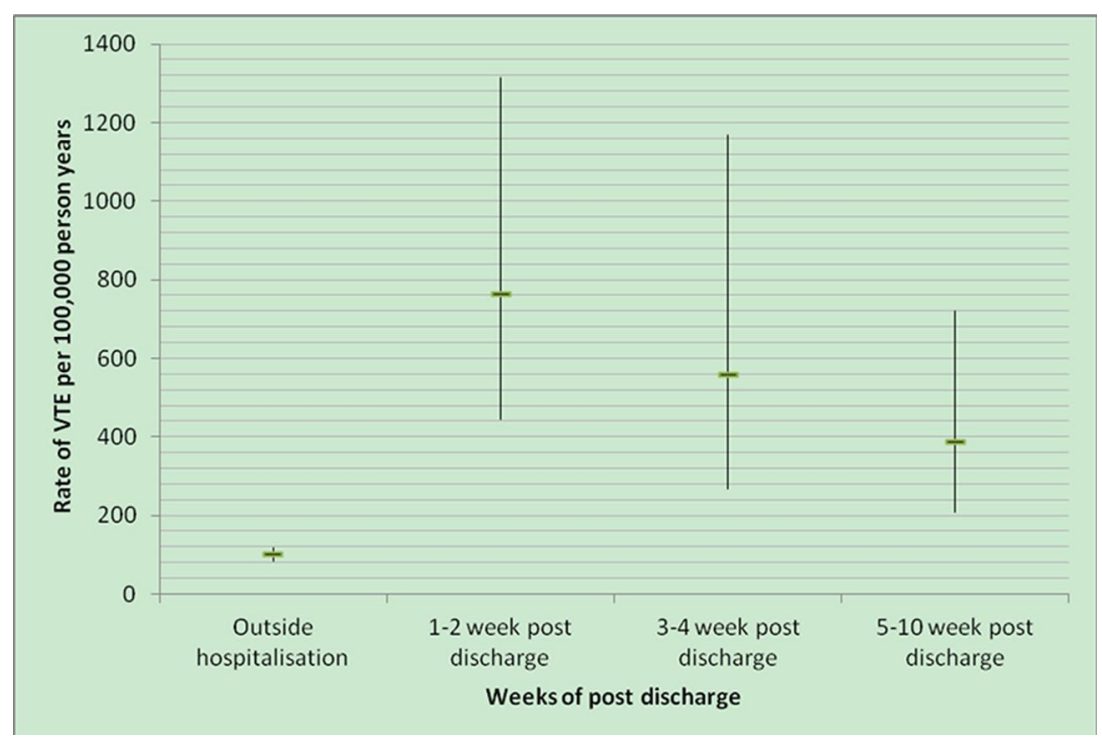

Fig 2 Rate of venous thromboembolism per 100000 person years by weeks after discharge during antepartum period: 12 events in weeks 1-2 after discharge, 7 events in weeks 3-4 after discharge, and 12 events in weeks 5-10 after discharge 